\title{
Interpretation of Cluster WBD frequency conversion mode data
}

\author{
J. S. Pickett, I. W. Christopher, and D. L. Kirchner \\ Department of Physics and Astronomy, The University of Iowa, Iowa City, Iowa 52242, USA \\ Correspondence to: J. S. Pickett (pickett@uiowa.edu)
}

Received: 31 May 2013 - Published in Geosci. Instrum. Method. Data Syst. Discuss.: 29 August 2013

Revised: 30 January 2014 - Accepted: 4 February 2014 - Published: 13 February 2014

\begin{abstract}
The Cluster wide-band data (WBD) plasma wave receiver mounted on each of the four Cluster spacecraft obtains high time resolution waveform data in the frequency range of $\sim 70 \mathrm{~Hz}$ to $577 \mathrm{kHz}$. In order to make measurements above $77 \mathrm{kHz}$, it uses frequency conversion to sample the higher frequency waves at one of three different conversion frequencies $(\sim 125,250$ and $500 \mathrm{kHz}$, these frequencies being the possible options for setting the base frequency of the frequency range being sampled) in one of three different filter bandwidths $(9.5,19$ and $77 \mathrm{kHz})$. Within the WBD instrument, a down-conversion technique, built around quadrature mixing, is used to convert these data to baseband $(0 \mathrm{kHz})$ in order to reduce the sample rate for telemetry to the ground. We describe this down-conversion technique and illustrate it through data obtained in space. Because these down-converted data sometimes contain pulses, which can be indicative of nonlinear physical structures (e.g., electron phase-space holes and electron density enhancements and depletions), it is necessary to understand what effects mixing and down conversion have on them. We present simulations using constructed signals containing pulses, nonlinear wave packets, sinusoids and noise. We show that the pulses and impulsive wave packets, if of sufficient amplitude and of appropriate width, survive the down-conversion process, sometimes with the same pulse shape but usually with reduced amplitude, and have timescales consistent with the filter bandwidth at the base frequency. Although we cannot infer the actual timescale of the pulses and impulsive wave packets as originally recorded by the WBD instrument before mixing and down conversion, their presence indicates nonlinear processes occurring at or somewhat near the location of the measurement. Sinusoidal waves are represented in the down-conversion timescale as sinusoids of nearly the same amplitude and at frequencies adjusted down by the conversion frequency. The original input waveforms, regardless
\end{abstract}

of their shape, whether pulses or sinusoids, can never be recovered from the down-converted waveforms.

\section{Introduction}

Each of the four spacecraft that comprise the ESA/NASA Cluster mission contains an identical wide-band data (WBD) plasma wave receiver, providing high time resolution waveform data from $\sim 70 \mathrm{~Hz}$ up to $577 \mathrm{kHz}$ (Gurnett et al., 1997). The waveform data obtained from these instruments are telemetered directly to ground stations, allowing for sample rates of $\sim 27-220 \mathrm{kHz}$ depending on the detection frequency bandwidth, $f_{\mathrm{BW}}(9.5,19$ or $77 \mathrm{kHz})$ and resolution $(1,4$ or 8 bit) modes that have been chosen. In order to obtain waveform data at frequencies higher than $77 \mathrm{kHz}$, the WBD instrument design allows for the input frequency range to be shifted by a frequency converter to one of four frequency ranges. The conversion frequency, $f_{0}$, determines the lower edge of the frequency range selected. The available values of $f_{0}$ are 0 (or baseband), 125,250 or $500 \mathrm{kHz}$ (approximate values, the exact values being provided in the Cluster WBD user guide archived at the Cluster Science Archive), so the digitized frequency range is then $f_{0}$ to $f_{0}+f_{\mathrm{BW}}$. Both the sampling frequency and $f_{0}$ are obtained by division from a $14 \mathrm{MHz}$ reference oscillator contained within the WBD instrument. The oscillator is phase-locked to the spacecraft's $220.753 \mathrm{kHz}$ high-frequency clock to maintain stability and match the instrument data rate to the spacecraft. The spacecraft ultra-stable oscillator's operating frequency of $2^{22} \mathrm{~Hz}$ is divided by 19 to derive the high-frequency clock so that WBD data are sampled with phase coherence to spacecraft timing.

If baseband $\left(f_{0}=0\right)$ is selected, the down-conversion stage is bypassed and the WBD signal in the frequency range 


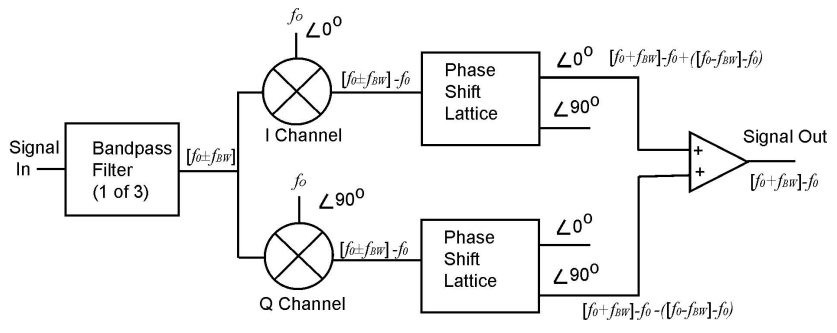

Fig. 1. Diagram showing the Cluster WBD frequency conversion chain for down converting a signal received at higher frequency $\left[f_{0}+f_{\mathrm{BW}}\right]$ to one at baseband frequency $\left(f_{0}=0\right)$.

0 to $f_{\mathrm{BW}}$ is routed directly to the digitizer with no frequency conversion. When $f_{0} \neq 0$, frequency translation is used to convert the input signal $\left[f_{0} \pm f_{\mathrm{BW}}\right]$ to baseband (see Fig. 1). Note that hereinafter brackets will be used to indicate a range of frequencies, either a double sideband range [ $\left.f_{0} \pm f_{\mathrm{BW}}\right]\left(f_{0}-f_{\mathrm{BW}}\right.$ to $\left.f_{0}+f_{\mathrm{BW}}\right)$ or a single sideband range such as $\left[f_{0}-f_{\mathrm{BW}}\right]\left(f_{0}-f_{\mathrm{BW}}\right.$ to $\left.f_{0}\right)$. Since only the upper sideband $\left[f_{0}+f_{\mathrm{BW}}\right]$ is the desired output, a quadrature mixer is used for single sideband detection. The quadrature mixer multiplies the input signal by $f_{0}$ in two channels, one using $f_{0}$ (the "I" or "In-phase" channel) and the other using $f_{0}$ phase shifted by $90^{\circ}$ (the "Q" or "Quadrature phase" channel). The two resulting baseband signals each contain the frequency range $\left[f_{0} \pm f_{\mathrm{BW}}\right]$. To cancel the unwanted sideband $\left[f_{0}-f_{\mathrm{BW}}\right]$, both channels are processed through two highprecision passive phase shift networks. The phase shift lattice is a three port device, with one input and two outputs, which differ from each other by $90^{\circ}$. In the I channel, the $0^{\circ}$ output is selected, while in the Q channel, the $90^{\circ}$ output is used. The $90^{\circ}$ phase shift added to the Q channel output shifts the positive sideband $\left[f_{0}+f_{\mathrm{BW}}\right]$ into phase coherence with the $\left[f_{0}+f_{\mathrm{BW}}\right]$ signal in the I channel, while the same shift moves the lower sideband $\left[f_{0}-f_{\mathrm{BW}}\right]$ into interference with the $\left[f_{0}-f_{\mathrm{BW}}\right]$ signal in the I channel. Summing the two selected outputs then cancels the unwanted lower sideband (Norgaard, 1956). The lower sideband could be selected by differencing the two outputs. The high-precision network allows for sideband cancellation exceeding the $48 \mathrm{~dB}$ capability of the 8 bit A/D converter. This technique allows for a significant reduction in cost and mass over the alternative procedure of procuring filters with sufficient selectivity to eliminate the unwanted lower sideband.

The down-conversion process preserves the frequency information in the signal, but it does not necessarily preserve the shape of the waveform, particularly when the frequency content of the signal is significantly filtered during bandpass filtering. Since the process is inherently Fourier in nature, pulses and other non-sinusoidal wave features may well be distorted. In order to better understand what happens to pulses undergoing this process, and thus how to interpret the results, we have simulated the mixing and down-conversion process that takes place within the WBD instrument by creating an artificial signal similar to what is observed by WBD in space.

The primary reason for requiring frequency conversion is to enable detection of high-frequency radio waves where direct sampling would exceed the allowable data rate to the spacecraft. The down-conversion process that takes place onboard within the WBD instrument allows for the selected detection band [ $f_{0}$ to $f_{\mathrm{BW}}$ ] to be shifted down in frequency to baseband and in so doing reduce the needed sample rate to one that fits the spacecraft telemetry system allocation. For example, in the case of the WBD $f_{0}=125 \mathrm{kHz}$ conversion mode using the $77 \mathrm{kHz}$ filter bandwidth, the unconverted input signal would need to be sampled at a minimum of $404 \mathrm{kbits} \mathrm{s}^{-1}$. The transmitter on the Cluster spacecraft can transmit the signal at a maximum of $262.144 \mathrm{kbits} \mathrm{s}^{-1}$. Continuous sampling can only be accomplished through the down-conversion process within the WBD instrument, which reduces the data volume before it is presented to the onboard data system for transmission to the ground.

Below we provide an example event during the Cluster mission showing data from a frequency conversion setting other than baseband and explain how the waveform data are processed on the ground. We then describe our method for simulating the down-conversion process in order to further help us determine how to interpret impulsive data, i.e., pulses and nonlinear wave packets that contain a wide range of frequencies. We conclude with a summary of our results and a guide for the interpretation of pulses obtained in space by instruments such as WBD when operating in conversion mode.

\section{Conversion mode data obtained in space}

The four-spacecraft Cluster mission was launched in 2000 with mission operations beginning in February 2001 following a 5-month commissioning phase. Cluster's primary purpose was to explore Earth's boundary layers with multi-point measurements in an approximate $4 \times 19 R_{\mathrm{E}}$ orbit (hereafter all references to $R_{\mathrm{E}}$ refer to geocentric distance). The WBD data provide the opportunity to study the waves in these boundary layers at their source of origin, as well as waves detected remotely well away from their source. The latter is carried out using very long baseline interferometry (VLBI) to locate that source, particularly with respect to auroral kilometric radiation (AKR). In order to study AKR, it is necessary to use the conversion modes discussed in the introduction, since most $\mathrm{AKR}$ is generated at frequencies greater than $125 \mathrm{kHz}$. However, in 2008, the Cluster orbit perigee decreased enough in altitude such that the spacecraft began to directly visit the source region of AKR and of auroral acceleration (roughly 1.2-2.6 $R_{\mathrm{E}}$ ). Embedded within the conversion mode $\left(f_{0} \neq 0\right)$ waveform data obtained in this region were the sinusoidal signatures of the AKR, as well as very short time duration pulses that have not previously been 
discussed in the literature due to the limitations of most instrumentation to detect them on very short timescales.

Figure $2 \mathrm{~b}$ is a frequency-time spectrogram covering a period of $2 \mathrm{~s}$, during which time Cluster spacecraft 1 transited the auroral upward current region at $2.2 R_{\mathrm{E}}(\sim 7600 \mathrm{~km}$ altitude). These data were obtained by the WBD receiver on 24 January 2010 in the form of waveforms in the $125 \mathrm{kHz}$ conversion mode with a $77 \mathrm{kHz}$ filter bandwidth, with $2180 \mathrm{sam}$ ples being obtained over an approximate $10 \mathrm{~ms}$ period every $80 \mathrm{~ms}$. As described above, the data were down-converted to baseband within the instrument before being transmitted to the ground, and then were analyzed by first calibrating them to electric field units of $\mathrm{mV} \mathrm{m}^{-1}$. Then an FFT was applied to each set of 1090 samples, thereby obtaining the frequency components in the baseband frequency domain $(0-77 \mathrm{kHz})$ and with amplitudes in $\mathrm{mV}^{2} \mathrm{~m}^{-2} \mathrm{~Hz}^{-1}$. In order to put these data back into the frequency range in which they were obtained in space, as opposed to that of baseband as they were telemetered to the ground, we now add the conversion factor, $125 \mathrm{kHz}$, to the frequency label of each Fourier component, in essence up-converting to the measured frequency domain. The amplitudes at each frequency, represented via the color scale in Fig. 2b, are in units of electric field spectral density. Prominent in Fig. 2b are AKR emissions around $192 \mathrm{kHz}$. Also prominent in this figure is a broad band that covers all frequencies at around 03:46:25.9 UT.

Plotted in Fig. 2a is a $10 \mu$ s sample of the waveforms obtained during the time of the data shown in Fig. 2b. These data show a pulse at 03:46:25.962054 UT, which is the cause of the broad band observed in Fig. 2b since the Fourier transform of a pulse will contain a wide range of frequencies, depending on pulse shape and width. Also apparent in Fig. $2 b$ are the sinusoidal waves associated with the AKR around $192 \mathrm{kHz}$. A crude analysis of Fig. 2a shows that from 0.0045 to $0.0048 \mathrm{~s}$, or a period of $0.0003 \mathrm{~s}$, there are 20 wave periods. Dividing the number of wave periods by the time duration over which they are observed yields a frequency of $66.7 \mathrm{kHz}$. This makes it apparent that the timescale shown on the horizontal axis in Fig. 2a is that of baseband, since we know from Fig. $2 b$ that the AKR is actually observed around $192 \mathrm{kHz}$. Simply adding the conversion factor of $125 \mathrm{kHz}$ to the frequency of the AKR obtained with the baseband timescale $(66.7 \mathrm{kHz})$ yields the expected frequency of the AKR of $192 \mathrm{kHz}$.

The pulse observed in Fig. 2a has a time duration of $27 \mu \mathrm{s}$ (start of pulse rise from base field to end of pulse fall at base field). If we think of this as a one-period wave, the inverse time duration would give us a frequency of $37.037 \mathrm{kHz}$. Adding $125 \mathrm{kHz}$ to this frequency yields a frequency of $\sim 162 \mathrm{kHz}$ and thus fits within the sampled conversion frequency range of $125-202 \mathrm{kHz}$, and as such we are not surprised that the pulse survived the down-conversion process.

This now brings us to the problem at hand. What is the true characteristic timescale of the pulse? Can we actually view it as a one-period wave and thus determine its charac-

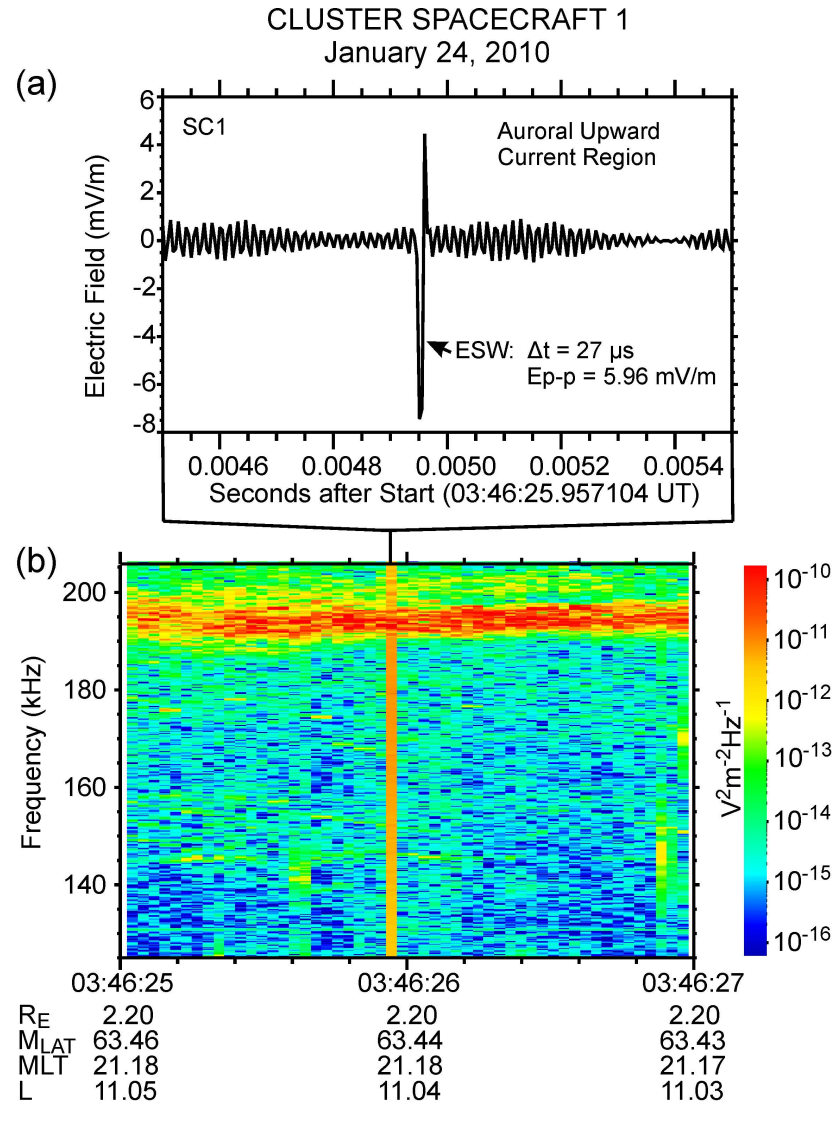

Fig. 2. WBD data obtained from Cluster 1 on 24 January 2010 in the auroral upward current region. (a) $1 \mathrm{~ms}$ duration waveform showing sinusoidal waves and a bipolar pulse. (b) $2 \mathrm{~s}$ frequency-time spectrogram showing that the sinusoidal waves represent AKR around $192 \mathrm{kHz}$ and the pulse represents the broad band observed just before $03: 46: 26 \mathrm{UT}$.

teristic time duration as it was sampled onboard before down conversion? Before down conversion, did the pulse actually have the form of a bipolar pulse (negative peak followed by a positive peak of similar amplitude, or vice versa), or did it possibly have some other embedded frequencies or structure that, after mixing and down conversion, produced the signature of a classical bipolar pulse (see, e.g., Pickett et al., 2004a)? To help answer these questions, we now look to the simulations.

\section{Simulations of conversion mode}

In order to simulate the mixing and down-conversion process that takes place within the WBD instrument, we first synthesized a very high time resolution digital waveform example and then performed the down conversion on this signal. 


\subsection{Constructed signal}

In seeking a form for our constructed signal, recall the previous section's discussion of the pulse found in the sample of actual WBD data. It was observed that if the pulse was thought of as a one-period wave, then the calculated "frequency" of the pulse $(37 \mathrm{kHz})$ was consistent with a $125 \mathrm{kHz}$ down conversion (the original "frequency" of the pulse was calculated as $162 \mathrm{kHz}$, which is within the 125 to $202 \mathrm{kHz}$ range of the conversion band/bandwidth being used). Taking this thought one step further, we can think of the observed pulse as a narrow wave packet - so narrow in fact that the envelope of the packet contains only a single period of an approximately pure sinusoid, which we call the carrier wave. For discussion, let us say that the packet is $30 \mu \mathrm{s}$ in length. Since the goal of the down-conversion process is in essence to preserve the envelope of waveforms (i.e., the information content of the waveform), while shifting the carrier frequency lower, we can presume that the original (preconversion) waveform also contained a $30 \mu$ s wave packet, but with a carrier wave frequency around $162 \mathrm{kHz}$, as calculated above. We take this to be the starting point for our simulations.

We construct a Gaussian wave packet of the following form:

$P(t)=A \sin (2 \pi F t+\varphi) e^{-(t / \tau)^{2}}$,

where $A$ is the packet amplitude, $F$ and $\varphi$ are the frequency and phase of the carrier wave, respectively, and $\tau=W / 3 \times 10^{6}$ ( $W$ is the width parameter, which sets the approximate width of the packet, in microseconds). A packet using $A=10, W=30 \mu \mathrm{s}, F=162 \mathrm{kHz}$ and $\varphi=0$ is shown in Fig. 3a, and the Fourier transform of this packet is shown in Fig. 4a. Note that much of the spectral energy of this waveform is contained within our conversion band (125 to $202 \mathrm{kHz}$ ), which gives us confidence that much of the information contained in the packet will survive the downconversion process.

\subsection{Down-converted signal}

The down-conversion process, performed via analog quadrature mixing circuitry in the instrument, is now applied to our constructed signal. The down conversion from a base of $125 \mathrm{kHz}$ to baseband of $0 \mathrm{kHz}$ is simulated by doing the quadrature mixing with a Hilbert transform (HT). This mixing could also have been simulated by an FIR (finite impulse response) filter, but we have chosen to use an HT since it is inherently simpler, while creating an FIR filter to deal with our complex signals can be quite difficult to achieve.

Figure $3 \mathrm{~b}$ shows the waveform from Fig. $3 \mathrm{a}$ after it has been down-converted using the above process. The result is a signature very like a bipolar pulse, with an amplitude that is close to $80 \%$ of the input amplitude and a width that is almost exactly the same as that of the input packet. Measur-
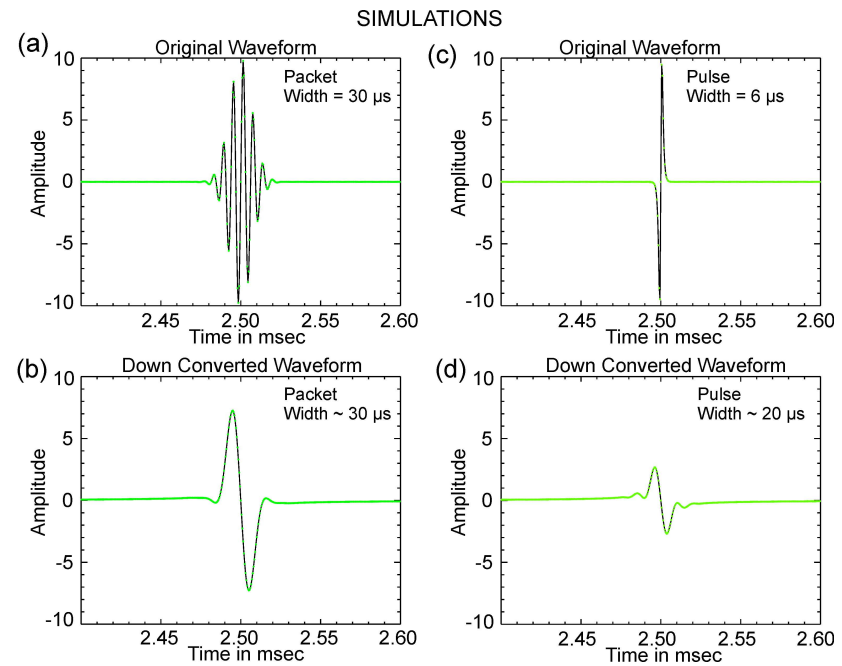

Fig. 3. Simulated waveform data both before and after down conversion from $125 \mathrm{kHz}$ using a $77 \mathrm{kHz}$ filter bandwidth for two types of input waves. (a) $0.2 \mathrm{~ms}$ portion of the input waveform consisting of a packet of duration $30 \mu \mathrm{s}$, encapsulating a $162 \mathrm{kHz}$ carrier wave. (b) Waveform data from (a) after down conversion, showing the waveform packet as a bipolar type pulse of duration $\sim 30 \mu$ s. (c) $0.2 \mathrm{~ms}$ portion of the input waveform consisting of a bipolar pulse of duration $6 \mu \mathrm{s}$. (d) Waveform data from (c) after down conversion, showing the output as a modified bipolar pulse of duration $\sim 20 \mu$ s.

ing the period of the sinusoid, we see that it is slightly under $30 \mu \mathrm{s}$, the width of the original packet, giving a frequency of $\sim 35 \mathrm{kHz}$. Adding $125 \mathrm{kHz}$ to this recovers the original carrier frequency of $162 \mathrm{kHz}$, which is within our measurement uncertainty. We thus confirm that a wave packet, at least in some circumstances, will be down-converted into a waveform that resembles a bipolar pulse.

\subsection{Other constructed waveforms}

The next question to ask is whether this is the only initial waveform that could result in such a bipolar output after down conversion. Figure 4a showed the spectral energy that is necessary to produce the down-converted waveform seen in Fig. 3b, but if we recall that the down-conversion process is designed to discard all spectral energy outside of the band (as well as down-shifting the energy within the band), then any spectral signature that fills much or all of the 125 to $202 \mathrm{kHz}$ band may do the same.

Consider now an input waveform that is an actual, very narrow, bipolar pulse, such as the $6 \mu$ s pulse shown in Fig. 3c. This pulse is defined by

$P(F)=E\left(\tanh \left(\frac{T}{\tau / 100}\right) / \cosh \left(\frac{T}{\tau / 100)}\right)\right) e^{-(T / \tau)^{2}}$,

where $E=2 \mathrm{AP}$ ( $A$ is the amplitude and $P= \pm 1$ sets the polarity of the pulse) and $\tau=W / 80000$. The spectral profile of this pulse is the black line shown in Fig. $4 \mathrm{~b}$, which is very 
SIMULATION SPECTRAL PROFILES

(a)

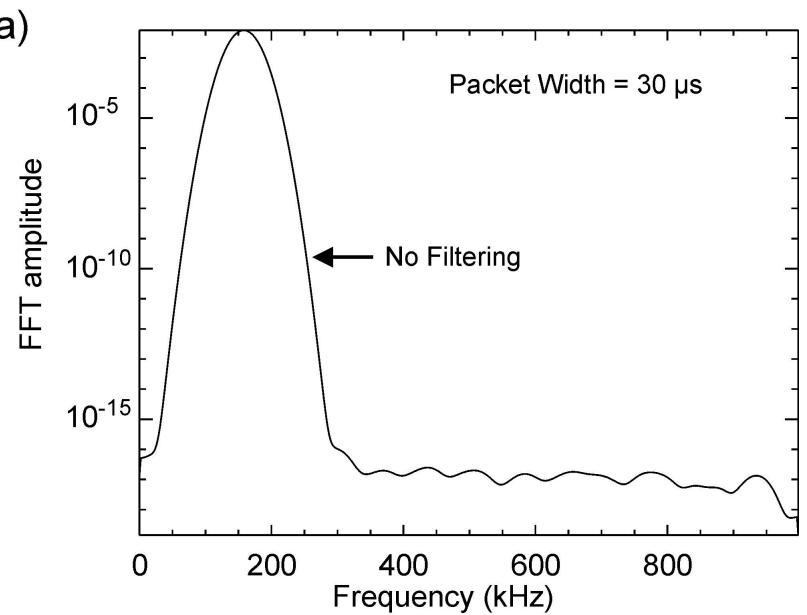

(b)

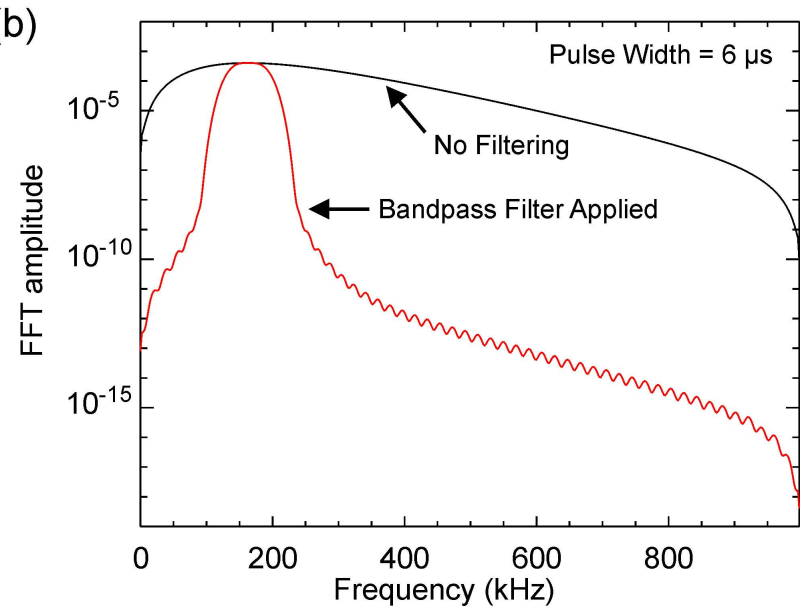

Fig. 4. Spectral profiles (amplitude vs. frequency) of simulated waveform data from Fig. 3 before down conversion. (a) Spectrum of the waveform packet presented in Fig. 3a showing a spectral peak at about $160 \mathrm{kHz}$, the energy being almost fully contained within the 125-202 kHz frequency band. (b) Black line: spectrum of the pulse presented in Fig. 3c showing a broad band nearly covering the entire Fourier frequency space, with much of the energy outside the 125-202 kHz frequency band. Red line: spectrum of the pulse after filtering down to the $125-202 \mathrm{kHz}$ frequency band to show comparison with the profile in Fig. $4 \mathrm{a}$.

broad, as expected, given the very short, sharp nature of the pulse. If we were to apply a 125 to $202 \mathrm{kHz}$ bandpass filter to this waveform (thus estimating the spectral energy that would survive down conversion), the energy that remains is shown by the red curve, which is very similar to, though somewhat narrower than, the profile seen in Fig. 4a. Encouraged by this, we apply our full down-conversion process to the pulse, with the result shown in Fig. 3d. The output is in fact a bipolar pulse, although a weakened and somewhat distorted one. There is, however, no obvious connection between the width of input and output pulses. This lack of connection is quite expected, though, since only a fraction of the initial pulse's spectral energy (the red curve vs. the black curve in Fig. 4b) will survive the down-conversion process. All of that lost energy, especially the high-frequency components, are key to defining the short, sharp initial pulse. The actual shape of the down-converted pulse in this case has much more to do with the width of the conversion band, and the precise details of the filters used to define it, than with the details of the input pulse.

Repeating this simulation for a range of input pulses shows similar results for widths of up to $\sim 30 \mu$ s. For widths longer than that, the spectral profiles of the pulses contain very little energy above $125 \mathrm{kHz}$, so almost nothing survives the downconversion process. Conversely, pulses longer than $\sim 30 \mu \mathrm{s}$ contain most of their energy in the baseband ( 0 to $77 \mathrm{kHz}$ ) of the WBD instrument, which is why such pulses are readily observed when the instrument is in that mode (e.g., Pickett et al., 2004a).

Finally, we have constructed a waveform that could simulate observations in space, similar to that shown in Fig. 2a. This waveform consists of three components: (1) low amplitude, approximately random background noise (RMS amplitude 0.29); (2) a few mid-amplitude pure sinusoids, both within, and above and below the desired conversion band (frequencies of 85, 138 and $150 \mathrm{kHz}$ with amplitudes of 2.1, 4.8 and 0.6 a.u. (arbitrary units), respectively); and (3) a high-amplitude solitary pulse (with width $5 \mu$ s and amplitude 10 a.u.) as per Eq. (2). We have chosen to simulate a solitary pulse similar to that in Fig. 3c, as opposed to the wave packet of Fig. 3a, because solitary pulses of this type (bipolar type structure; see Pickett et al., 2004a, b) are observed very frequently in the space data obtained in baseband $\left(f_{0}=0\right.$, i.e., no down conversion) with timescales only slightly longer than our simulated pulse before down conversion. Figure 5a shows a $200 \mu$ s sample taken from the constructed waveform (whose full length is $5 \mathrm{~ms}$ ) around the time of the injected pulse. A pulse of $5 \mu$ s in duration is seen in the middle of the sample, at $t=100 \mu \mathrm{s}$, with an amplitude of 10 a.u., while the background noise and other sinusoidal signals are seen throughout, centered at 0 amplitude with a net amplitude of 1-1.5 a.u. An FFT of the full constructed waveform is shown in Fig. 5b. This frequency-time spectrogram covers a period of about $5 \mathrm{~ms}$, which is comparable to a single frame obtained by the WBD instrument using the $77 \mathrm{kHz}$ filter bandwidth with a $125 \mathrm{kHz}$ conversion. Here we clearly see two sinusoidal waves showing up with medium amplitudes around 140 and $150 \mathrm{kHz}$, similar to AKR frequencies, as well as some weaker waves at and above $175 \mathrm{kHz}$, throughout the plot. The single injected pulse is observed as a broad band around $0.0023 \mathrm{~s}$.

Figure $5 \mathrm{c}$ is the same $5 \mathrm{~ms}$ constructed waveform as that seen in Fig. 5a with the exception that it has been mixed and down-converted using the HT. We can immediately see the effects of this process in the waveform. The sinusoidal waves and background noise have been smoothed out, and 


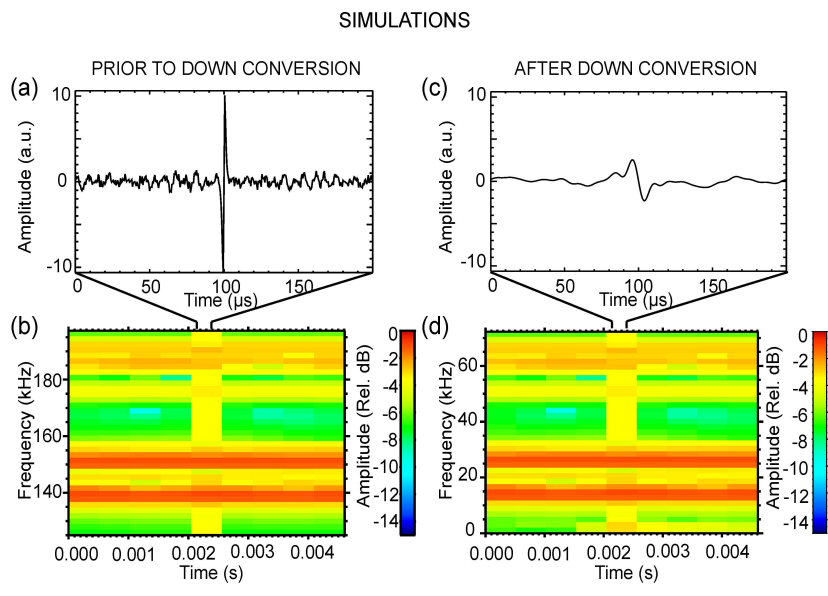

Fig. 5. Simulated data both before and after down conversion for comparison to space observations shown in Fig. 2. (a) $200 \mu$ s sample of the input waveform showing the $5 \mu$ constructed pulse, as well as sinusoidal oscillations and background noise, prior to down conversion. (b) $5 \mathrm{~ms}$ time-frequency spectrogram of the simulated data shown in (a). (c) $200 \mu$ s sample of the waveform presented in (a), after having been down-converted from the frequency range 125$202 \mathrm{kHz}$ to baseband; the sinusoidal waves have been smoothed out and time dilated by the process, and the pulse has been reduced in amplitude, and broadened in time, consistent with the effects of the $77 \mathrm{kHz}$ band filter. (d) $5 \mathrm{~ms}$ time-frequency spectrogram of the down-converted waveform in (c) showing the broad band associated with the pulse, as well as the sinusoidal waves on the downconverted frequency scale.

the pulse has been greatly reduced in amplitude (now about 2.5 a.u.) and stretched out in time (time duration now about $15 \mu \mathrm{s})$. However, the stretch out in time is consistent with the filter bandwidth $(77 \mathrm{kHz})$ and is expected for the pulse as discussed earlier in this section. Because of the decreased amplitude, it is not possible to determine the period of the sinusoidal waves, because we have down-converted to the timescale of baseband ( $0 \mathrm{kHz}$ base frequency).

Figure 5d thus shows an FFT spectrogram of the waveform seen in Fig. 5c now with a frequency range from 0 to $77 \mathrm{kHz}$. When we compare this spectrogram to the one prior to down conversion (Fig. 5b), we see that all of the frequency information has been precisely preserved, including the pulse, and that no frequency content from outside our desired band has been shifted into the band. However, some low-frequency noise at the bottom of the frequency band has been artificially introduced during the down-conversion process.

\section{Interpretation and guidance}

Several waveform data samples containing noise, sinusoidal waves and pulses were constructed as discussed in Sect. 3. Using an HT to mix and down-convert the constructed waveforms to a base frequency of $0 \mathrm{kHz}$, we have demonstrated that (1) sinusoidal waves will be represented as sine waves with periods consistent with the original frequency shifted down by the conversion factor (in our case $125 \mathrm{kHz}$ ), and (2) pulses and wave packet bursts with sufficient amplitudes will be down-converted to the base frequency with timescales that to a large degree result from the filter bandwidth that was selected (for example, inverting $77 \mathrm{kHz}$ gives a timescale of $\sim 13 \mu \mathrm{s})$. We can thus generalize the results obtained in Sect. 3 and apply them to the Cluster WBD data by saying that if the incident waveform contains any short (less than 20-30 $\mu$ s in duration), intense (relative to any other ambient signal) pulse, of any particular shape, then it will have a broad spectral profile. Such a profile will likely fill the Cluster WBD 125 to $202 \mathrm{kHz}$ frequency band with energy, and thus will result in a bipolar-like structure (specifically roughly one period of a sinusoidal wave packet) in the downconverted waveform. These bipolar-like structures are likely to have apparent widths ranging from $\sim 30 \mu$ s down to the digitization limit of the instrument $(15-20 \mu \mathrm{s})$. Note that if the input pulse is too short, less than a microsecond or so, then much of the spectral energy has shifted above $202 \mathrm{kHz}$, so again there will not be much energy that survives the down-conversion process. At the other end of the timescale, it should be noted that the multipole filters used within the WBD instrument to select each of the three filter bandwidths can distort pulses that are too long in duration (see Swanner et al., 2006; also included as supplementary material with this article). As discussed above, though, such pulses would only be observed in data taken in baseband mode.

In contrast to pulse-like structures, sinusoidal waves with frequencies that fall within the selected conversion band will be down-converted to base frequency with nearly $100 \%$ of their original energy retained, as per the design of the WBD instrument. The apparent frequency of these waves, when seen as waveforms, will be that of their original frequency minus the conversion $(\sim 125,250$ or $500 \mathrm{kHz})$. Their true (i.e., original) frequencies are easily recovered after transforming the waveforms into the frequency domain (via FFT) by upshifting each displayed frequency by that same amount.

Historically, broad band signals have often been seen in the spectrograms of AKR as observed from space (such as that seen in Fig. 2). Most of those data were obtained in the spectral domain. Thus, there was no way to determine the source of those broad bands. With the advent of waveform receivers with high sampling rates, we have learned that those broad bands, at least when observed at the base frequency, often are indicative of electrostatic structures, such as electron phase-space holes, that are created out of nonlinear processes (e.g., Pickett et al., 2004a, b). Knowing now that similar broad band signals in conversion mode data could possibly indicate the presence of similar type structures, or impulsive, nonlinear type wave packets, we can now more effectively analyze AKR data obtained in the auroral acceleration region, or in any other magnetospheric region. By noting their presence, we can use this knowledge when analyzing 
the generation of AKR, particularly its fine structure, and other auroral processes.

As a final note, it may be that more in-depth illumination of the complications involved in interpreting pulse-like structures in conversion mode data could be obtained from the field of astronomy. Astronomers have typically used similar and more complex methods in analyzing data from pulsars where the time periods of the signal pulse received from the pulsar is so short that sampling of the signal must take place at very high rates with subsequent down conversion (see, e.g., Hankins and Rickett, 1975). It is possible that a study of these techniques could lead to methods that would allow more to be learned about the pulses and/or impulsive wave packets that are the source of the structures and broad band signals seen in the WBD conversion mode data. However, we stress that the original waveforms, whether input pulses and wave packets or pure sinusoids, are never preserved or recorded by WBD when using down conversion. Thus the exact structure of the original input waveform can never be known or recovered from the down-converted waveform from Cluster WBD or from any similar waveform instrument using downconversion techniques.

\section{Supplementary material related to this article is available online at http://www.geosci-instrum-method-data-syst.net/3/21/ 2014/gi-3-21-2014-supplement.pdf.}

Acknowledgements. We acknowledge support from NASA Goddard Space Flight Center under grants NNX11AB38G and NNX14AB10G. We also acknowledge fruitful discussions with R. L. Huff and J. R. Phillips from the University of Iowa in helping us to understand the frequency conversion mode through bench tests with the flight spare WBD receiver.

Edited by: H. Laakso

\section{References}

Gurnett, D. A., Huff, R. L., and Kirchner, D. L.: The Wide-Band Plasma Wave Investigation, Space Sci. Rev., 79, 195-208, 1997.

Hankins, T. H. and Rickett, B. J.: Pulsar signal processing, Meth. Comp. Phys. 14, 55-129, 1975.

Norgaard, E. E.: The phase-shift method of single-sideband signal generation, Proc. IRE, 44, 1735-1743, 1956.

Pickett, J. S., Chen, L.-J., Kahler, S. W., Santolík, O., Gurnett, D. A., Tsurutani, B. T., and Balogh, A.: Isolated electrostatic structures observed throughout the Cluster orbit: relationship to magnetic field strength, Ann. Geophys., 22, 2515-2523, doi:10.5194/angeo-22-2515-2004, 2004a.

Pickett, J. S., Kahler, S. W., Chen, L.-J., Huff, R. L., Santolík, O., Khotyaintsev, Y., Décréau, P. M. E., Winningham, D. Frahm, R., Goldstein, M. L., Lakhina, G. S., Tsurutani, B. T., Lavraud, B., Gurnett, D. A., André, M., Fazakerley, A., Balogh, A., and Rème, H.: Solitary waves observed in the auroral zone: the Cluster multi-spacecraft perspective, Nonlinear Proc. Geoph., 11, 183196, 2004b.

Swanner, J. M., Pickett, J. S., Phillips, D. L., and Kirchner, D. L.: WBD response to bipolar and tripolar pulses: bench tests vs. in flight observations, available at: www-pw.physics.uiowa.edu/ cluster/pulse_tests.pdf and www.rssd.esa.int/index.php?project= CLUSTER\&page $=$ documentation (last access for both: 8 February 2014), 2006. 